Supporting Information

\title{
Enhanced Structural Stability and Volumetric Capacity of 3D Pyknotic Graphene Conductive Network via Pillar Effect of Sn Nanoparticles for Sodium-ion Batteries
}

Hongbao Li, ${ }^{1}$ Zhenli Fu ${ }^{1}$, Hongwei Kang ${ }^{3}$, Rui Wang ${ }^{1}$, Rong Hua ${ }^{1}$, Quanwei Ma ${ }^{1}$, Longhai Zhang $^{1,2 *}$, Chaofeng Zhang ${ }^{1,2}$, and Tengfei Zhoul*

1 Institutes of Physical Science and Information Technology, Information Materials and Intelligent Sensing Laboratory of Anhui Province, Leibniz Joint Research Center of Materials Sciences, Engineering Laboratory of High Performance Waterborne Polymer Materials of Anhui Province, Anhui Graphene Engineering Laboratory, Key Laboratory of Structure and Functional Regulation of Hybrid Material (Ministry of Education), Anhui University, Hefei 230601, China

2 Key Laboratory of Advanced Energy Materials Chemistry (Ministry of Education), College of Chemistry, Nankai University, Tianjin 300071, China

3 School of Chemistry and Materials Engineering, Engineering Research Center of Biomass Conversion and Pollution Prevention of Anhui Educational Institutions, Fuyang Normal University, Fuyang 236037, China

H.B. Li and Z.L Fu contributed equally to this work.

*Corresponding author

E-mail:tengfeiz@ahu.edu.cn,zlhedu@163.com 


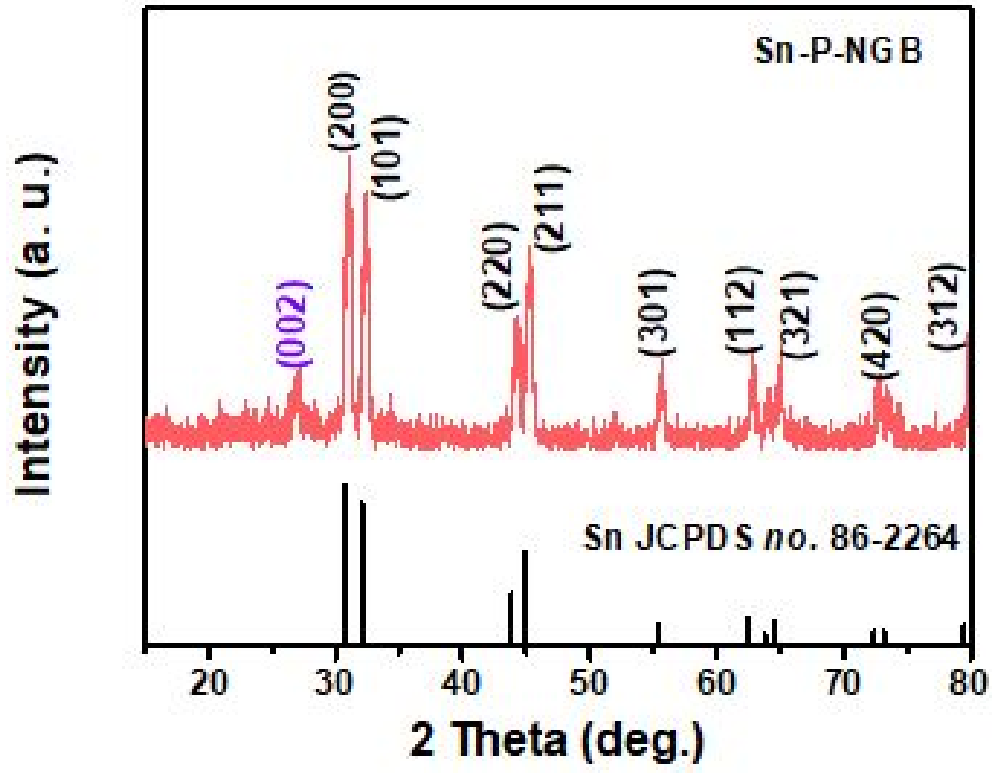

Figure S1. XRD pattern of the Sn-P-NGB.

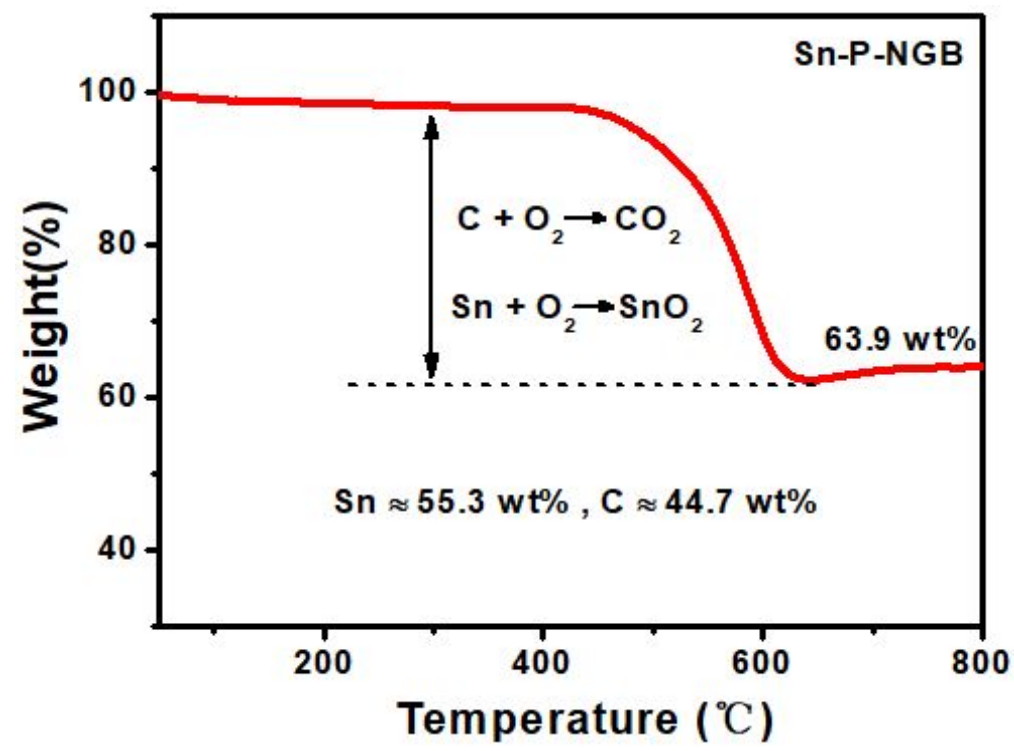

Figure S2. TG curve of the Sn-P-NGB. 


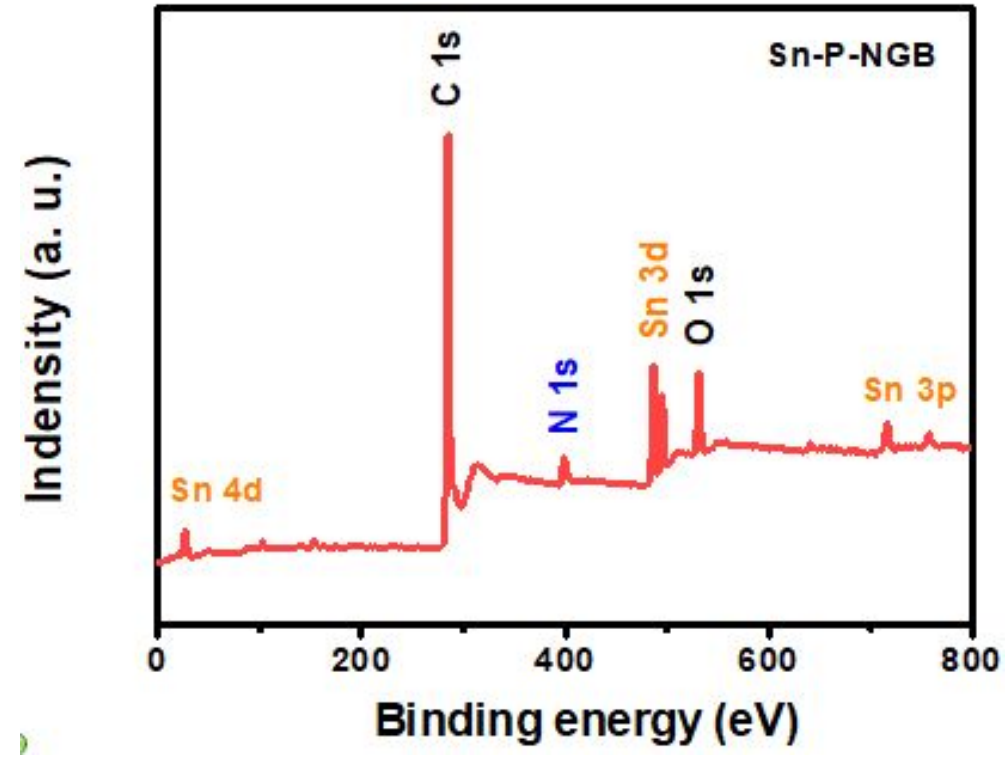

Figure S3. XPS survey spectra of Sn-P-NGB.

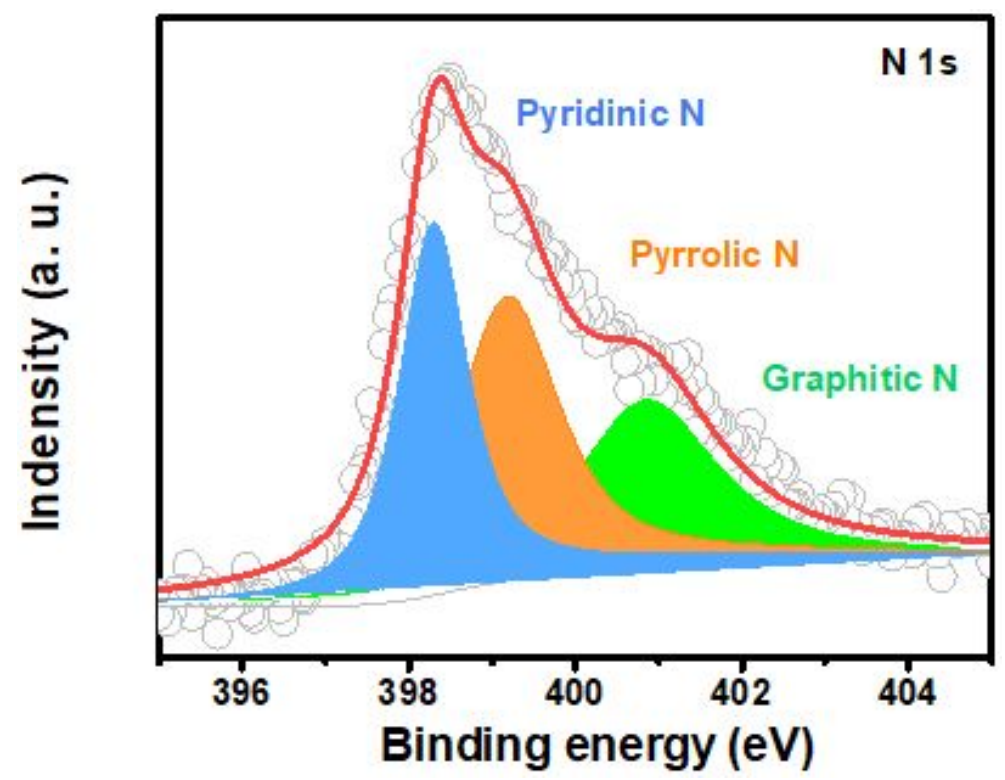

Figure S4. High-resolution N 1s spectrum of Sn-P-NGB. 
Table S1. Comparison of the results with other reported anodes for SIBs.

\begin{tabular}{|c|c|c|c|c|}
\hline Sample & Rate capability & Rate capability & Mass loading & Ref. \\
\hline \multirow{3}{*}{$\mathrm{Sb} / \mathrm{C}$ composite } & $\operatorname{Rate}_{(\min )}: 0.1 \mathrm{~A} \mathrm{~g}^{-1}$ & $\operatorname{Rate}_{(\max )}: 2 \mathrm{~A} \mathrm{~g} \mathrm{~g}^{-1}$ & \multirow{3}{*}{$1.3-1.5 \mathrm{mg} \mathrm{cm}^{-2}$} & \multirow{3}{*}{ [1] } \\
\hline & & & & \\
\hline & Capacity: $421 \mathrm{mAh} \mathrm{cm}{ }^{-3}$ & Capacity: $376 \mathrm{mAh} \mathrm{cm}^{-3}$ & & \\
\hline \multirow{3}{*}{ Graphene block } & $\operatorname{Rate}_{(\min )}: 0.02$ A g $^{-1}$ & & \multirow{3}{*}{$1.5 \mathrm{mg} \mathrm{cm}^{-2}$} & \multirow{3}{*}{ [2] } \\
\hline & & - & & \\
\hline & Capacity: $294.3 \mathrm{mAh} \mathrm{cm}^{-3}$ & & & \\
\hline \multirow{3}{*}{ N-CDGB } & $\operatorname{Rate}_{(\min )}: 0.02$ A g $^{-1}$ & $\operatorname{Rate}_{(\max )}: 10 \mathrm{~A} \mathrm{~g}^{-1}$ & \multirow{3}{*}{$\approx 1.5 \mathrm{mg} \mathrm{cm}^{-2}$} & \multirow{3}{*}{ [2] } \\
\hline & & & & \\
\hline & Capacity: $780 \mathrm{mAh} \mathrm{cm}^{-3}$ & Capacity: $177 \mathrm{mAh} \mathrm{cm}^{-3}$ & & \\
\hline \multirow{3}{*}{ Defective graphene } & $\operatorname{Rate}_{(\min )}: 0.05$ A g $^{-1}$ & $\operatorname{Rate}_{(\max )}: 10 \mathrm{~A} \mathrm{~g}^{-1}$ & \multirow{3}{*}{$2.5 \mathrm{mg} \mathrm{cm}^{-2}$} & \multirow{3}{*}[3]{} \\
\hline & & & & \\
\hline & Capacity: $397 \mathrm{mAh} \mathrm{cm}^{-3}$ & Capacity: $181 \mathrm{mAh} \mathrm{cm}^{-3}$ & & \\
\hline $\mathrm{SnS}_{2} /$ graphene & $\operatorname{Rate}_{(\min )}: 0.1 \mathrm{~A} \mathrm{~g}^{-1}$ & $\operatorname{Rate}_{(\max )}: 10 \mathrm{~A} \mathrm{~g}^{-1}$ & \multirow[b]{2}{*}{$0.8 \mathrm{mg} \mathrm{cm}^{-2}$} & \multirow[b]{2}{*}{ [4] } \\
\hline \multirow[t]{2}{*}{ ribbon } & Capacity: $508 \mathrm{mAh} \mathrm{cm}^{-3}$ & Capacity: $244 \mathrm{mAh} \mathrm{cm}^{-3}$ & & \\
\hline & $\operatorname{Rate}_{(\min )}: 0.05 \mathrm{~A} \mathrm{~g}^{-1}$ & & & \\
\hline \multirow[t]{2}{*}{$\mathrm{SnO}_{2}$ nanosphere } & & - & - & {$[5]$} \\
\hline & Capacity: $970 \mathrm{mAh} \mathrm{cm}^{-3}$ & & & \\
\hline \multirow[t]{2}{*}{$\mathrm{Ti}_{3} \mathrm{C}_{2}$} & $\operatorname{Rate}_{(\min )}: 0.02 \mathrm{~A} \mathrm{~g} \mathrm{~g}^{-1}$ & $\operatorname{Rate}_{(\max )}: 5 \mathrm{~A} \mathrm{~g} \mathrm{~g}^{-1}$ & & \\
\hline & Capacitv: $421 \mathrm{mAh} \mathrm{cm}^{-3}$ & Capacitv: $89 \mathrm{mAh} \mathrm{cm}^{-3}$ & - & [6] \\
\hline \multirow[t]{2}{*}{ Ni-supported } & $\operatorname{Rate}_{(\min )}: 0.2 \mathrm{~A} \mathrm{~g}^{-1}$ & & & \\
\hline & & - & - & [7] \\
\hline \multirow[t]{2}{*}{$\mathrm{Sb}_{2} \mathrm{O}_{3}$} & Capacity: $488 \mathrm{mAh} \mathrm{cm}^{-3}$ & & & \\
\hline & $\operatorname{Rate}_{(\min )}: 0.1 \mathrm{~A} \mathrm{~g}^{-1}$ & $\operatorname{Rate}_{(\min )}: 5 \mathrm{~A} \mathrm{~g} \mathrm{~g}^{-1}$ & $\approx 1.5-1.8 \mathrm{mg}$ & This \\
\hline Sn-P-NGB & Capacity: $1462 \mathrm{mAh} \mathrm{cm}^{-3}$ & Capacity: $513 \mathrm{mAh} \mathrm{cm}^{-3}$ & $\mathrm{~cm}^{-2}$ & work \\
\hline
\end{tabular}




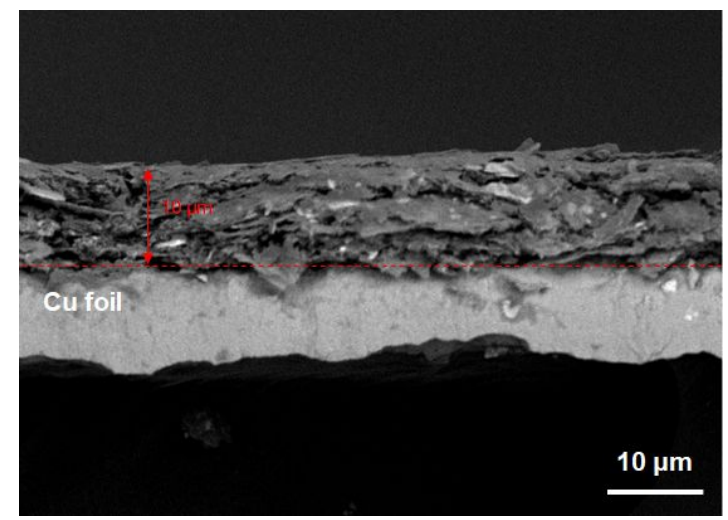

Figure S5. SEM cross-section image of the Sn-P-NGB electrode
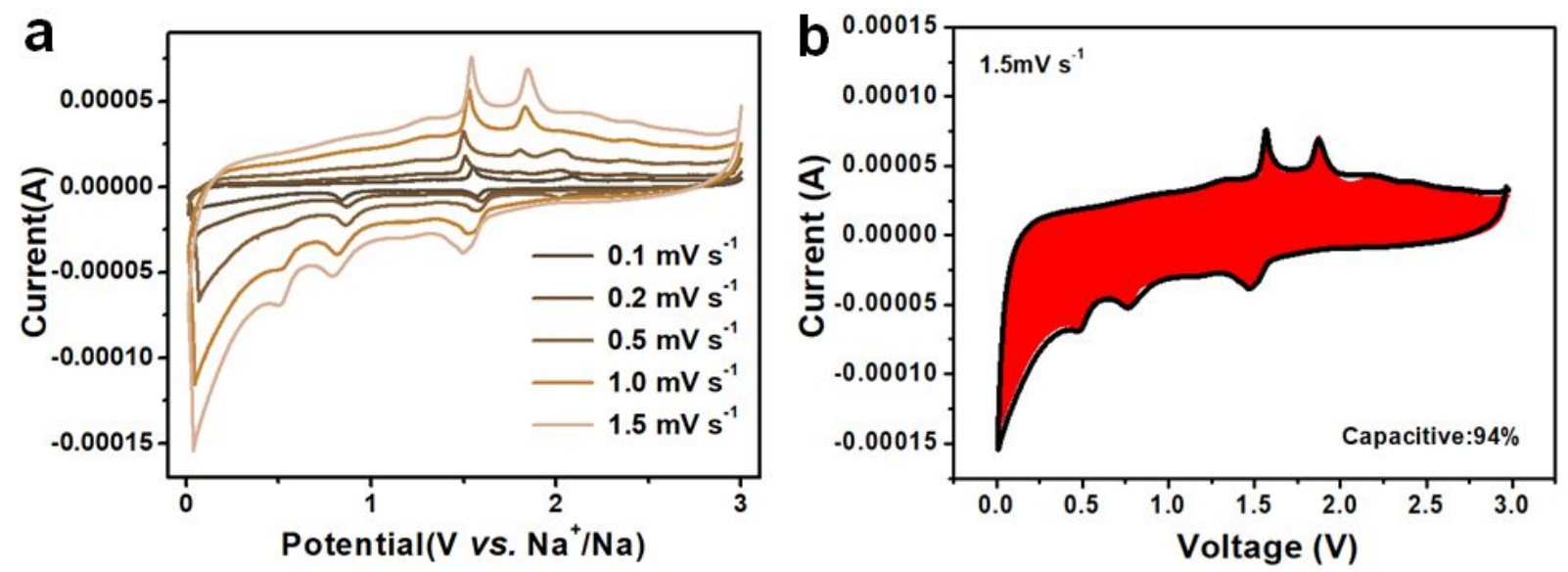

Figure S6. (a) CV curves of Sn-P-NGB anode at various sweep rates, and (b) corresponding capacitive contribution at $1.5 \mathrm{mV} \mathrm{s}^{-1}$ (red region). 


\section{References}

(1) Selvaraj, B.; Huang, S.-S.; Wu, C.-E.; Lin, Y.-H.; Wang, C.-C.; Song, Y.-F.; Lu, M.-L.;

Sheu, H.-S.; Wu, N.-L. Micrometer-Sized Nanoporous Sb/C Anode with High Volumetric Capacity and Fast Charging Performance for Sodium-Ion Batteries. ACS Applied Energy Materials 2018, 1, 2317-2325.

(2) Liu, Z.; Zhang, L.; Sheng, L.; Zhou, Q.; Wei, T.; Feng, J.; Fan, Z. Edge-Nitrogen-Rich Carbon Dots Pillared Graphene Blocks with Ultrahigh Volumetric/Gravimetric Capacities and Ultralong Life for Sodium-Ion Storage. Adv. Energy Mater. 2018, 8, 1802042.

(3) Dong, Y.; Lin, X.; Wang, D.; Yuan, R.; Zhang, S.; Chen, X.; Bulusheva, L. G.; Okotrub, A. V.; Song, H. Modulating The Defects of Graphene Blocks by Ball-Milling for Ultrahigh Gravimetric and Volumetric Performance and Fast Sodium Storage. Energy Storage Mater. 2020, $30,287-295$.

(4) Liu, Y.; Yang, Y.; Wang, X.; Dong, Y.; Tang, Y.; Yu, Z.; Zhao, Z.; Qiu, J. Flexible Paper-like Free-Standing Electrodes by Anchoring Ultrafine $\mathrm{SnS}_{2}$ Nanocrystals on Graphene Nanoribbons for High-Performance Sodium Ion Batteries. ACS Appl. Mater. Interfaces 2017, 9, 15484-15491.

(5) Lu, X.; Luo, F.; Xiong, Q.; Chi, H.; Qin, H.; Ji, Z.; Tong, L.; Pan, H. Sn-MOF Derived Bimodal-Distributed $\mathrm{SnO}_{2}$ Nanosphere as A High Performance Anode of Sodium Ion Batteries with High Gravimetric and Volumetric Capacities. Mater. Res. Bull. 2018, 99, 45-51.

(6) Xie, X.; Zhao, M.-Q.; Anasori, B.; Maleski, K.; Ren, C. E.; Li, J.; Byles, B. W.; Pomerantseva, E.; Wang, G.; Gogotsi, Y. Porous Heterostructured Mxene/Carbon Nanotube Composite Paper with High Volumetric Capacity for Sodium-Based Energy Storage Devices. Nano Energy 2016, 26, 513-523.

(7) Kim, S.; Qu, S.; Zhang, R.; Braun, P. V. High Volumetric and Gravimetric Capacity Electrodeposited Mesostructured $\mathrm{Sb}_{2} \mathrm{O}_{3}$ Sodium Ion Battery Anodes. Small 2019, 15, e1900258. 